\title{
Botulinum Toxin Type A Injection for Neuropathic Pain in a Patient With a Brain Tumor: A Case Report
}

\author{
Kyung Eun Nam, MD, Joon Sung Kim, MD, PhD, Bo Young Hong, MD, PhD, \\ Bomi Sul, MD, Hyehoon Choi, MD, So Yeon Jun, MD, Seong Hoon Lim, MD, PhD
}

Department of Rehabilitation Medicine, St. Vincent's Hospital, College of Medicine, The Catholic University of Korea, Suwon, Korea

\begin{abstract}
Neuropathic pain is usually managed pharmacologically, rather than with botulinum toxin type A (BTX-A). However, medications commonly fail to relieve pain effectively or have intolerable side effects. We present the case of a 62-year-old man diagnosed with an intracranial chondrosarcoma, which was removed surgically and treated with radiation therapy. He suffered from neuropathic pain despite combined pharmacological therapy with gabapentin, amitriptyline, tramadol, diazepam, and duloxetine because of adverse effects. BTX-A (100 units) was injected subcutaneously in the most painful area in the posterior left thigh. Immediately after the injection, his pain decreased significantly from $6 / 10$ to $2 / 10$ on a visual analogue scale. Pain relief lasted for 12 weeks. This case report describes intractable neuropathic pain caused by a brain tumor that was treated with subcutaneous BTX-A, which is a useful addition for the management of neuropathic pain related to a brain tumor.
\end{abstract}

Keywords Botulinum toxins, Neuralgia, Brain neoplasms

\section{INTRODUCTION}

The International Association for the Study of Pain defines neuropathic pain as "pain caused by a lesion or disease of the somatosensory system". Central neuropathic pain is a term used to describe pain caused by a direct insult to central nervous system (CNS) structures due to vas- cular, infectious, demyelinating, traumatic, or neoplastic disorders [1]. Management options for neuropathic pain include various pain medications and neuromodulation therapy. Still, pain treatment can be very difficult [1]. We report clinically meaningful improvement in neuropathic pain related to a brain tumor in a patient after subcutaneous injection of botulinum toxin type A (BTX-A).

Received June 14, 2017; Accepted July 18, 2017

Corresponding author: Seong Hoon Lim

Department of Rehabilitation Medicine, St. Vincent's Hospital, College of Medicine, The Catholic University of Korea, 93 Jungbu-daero, Paldal-gu, Suwon 16247, Korea. Tel: +82-31-249-7650, Fax: +82-31-251-4481, E-mail: seonghoon@catholic.ac.kr

ORCID: Kyung Eun Nam (https://orcid.org/0000-0001-5195-4320); Joon Sung Kim (https://orcid.org/0000-0001-7457-593X); Bo Young Hong (https:// orcid.org/0000-0001-9290-6173); Bomi Sul (https://orcid.org/0000-0001-7166-8997); Hyehoon Choi (https://orcid.org/0000-0002-1470-2914); So Yeon Jun (https://orcid.org/0000-0003-3217-1990); Seong Hoon Lim (https://orcid.org/0000-0002-5475-4153).

@ This is an open-access article distributed under the terms of the Creative Commons Attribution Non-Commercial License (http://creativecommons.org/ licenses/by-nc/4.0) which permits unrestricted noncommercial use, distribution, and reproduction in any medium, provided the original work is properly cited. Copyright $\odot 2017$ by Korean Academy of Rehabilitation Medicine 


\section{CASE REPORT}

We treated a 62-year-old man with a several-year history of neuropathic pain. In 2002, he developed dizziness and diplopia. Intracranial chondrosarcoma diagnosed after a biopsy of a tumor at the right cerebellopontine angle (medial clivus and petrous part of the temporal bone). He underwent CyberKnife radiosurgery in 2002, and a right suboccipital craniectomy and tumor removal due to regrowth of the tumor in 2004 at another hospital. Since then, he had experienced left hemiplegia with a hemisensory deficit, right facial palsy, right cranial nerve VI palsy, and dysphagia. He suffered from neuropathic pain manifest as an uncomfortable cold squeezing and tightness, with allodynia to light touch in the left hemibody, especially the posterior thigh.

He was referred to the Department of Neurosurgery at St. Vincent's Hospital for a neuromodulation technique to treat the pain. In 2008, deep brain stimulation (DBS) was performed using the right ventral posterolateral nucleus and periventricular grey matter as targets. After the DBS and medication, the pain disappeared. However, the DBS was discontinued in 2013 because he developed a headache, and he took only medication to treat the pain. There was no evidence suggesting tumor regrowth on follow-up magnetic resonance imaging (Fig. 1). Although the pain in the left posterior thigh gradually worsened, he could not take opioids (oxycodone, hydromorphone, or a fentanyl patch) or more than 1,200 mg of gabapentin because of side effects. He had been taking the maximum daily tolerable dosage of medication, a combination of gabapentin 1,200 mg, amitriptyline $10 \mathrm{mg}$, diazepam 4 $\mathrm{mg}$, tramadol $100 \mathrm{mg}$, and duloxetine $60 \mathrm{mg}$. The regimen was not sufficient to reduce his pain.

In August 2016, his neurological symptoms worsened and he developed hoarseness of voice due to a right vocal cord palsy caused by recurrence of the sarcoma (Fig. 2). In addition, his neuropathic pain worsened. He could not sit on a wheelchair because of severe pain. Together with his family and a physiatrist, the patient decided to see if the neuropathic pain could be managed with an injection of BTX-A, so that he could sit. BTX-A 100 units (Botox; Allergan, Irvine, CA, USA) was diluted in $2.4 \mathrm{~mL}$ normal saline. The preparation was injected subcutaneously in the most painful area of the posterior left thigh at 16 locations based on height and weight of $168 \mathrm{~cm}$ and $52 \mathrm{~kg}$, respectively (Fig. 3). No immediate side effects were seen after the injection. The day after the injection, his pain had decreased from $6 / 10$ before the injection to $2 / 10$ on a visual analogue scale (VAS). The pain was well-controlled (2-3/10 on the VAS) 12 weeks after the injection and he was satisfied with the BTX-A injection.

\section{DISCUSSION}

BTX-A injections have proven anti-nociceptive effects for neuropathic pain in some disorders, but have been used only experimentally. This case report is the first article to describe the analgesic effect of subcutaneous BTX-A in neuropathic pain related to a brain tumor. Pharmacological management remains the most important therapeutic option for neuropathic pain, and combining two or more different drugs has been reported to be more beneficial than monotherapy [1]. Many patients
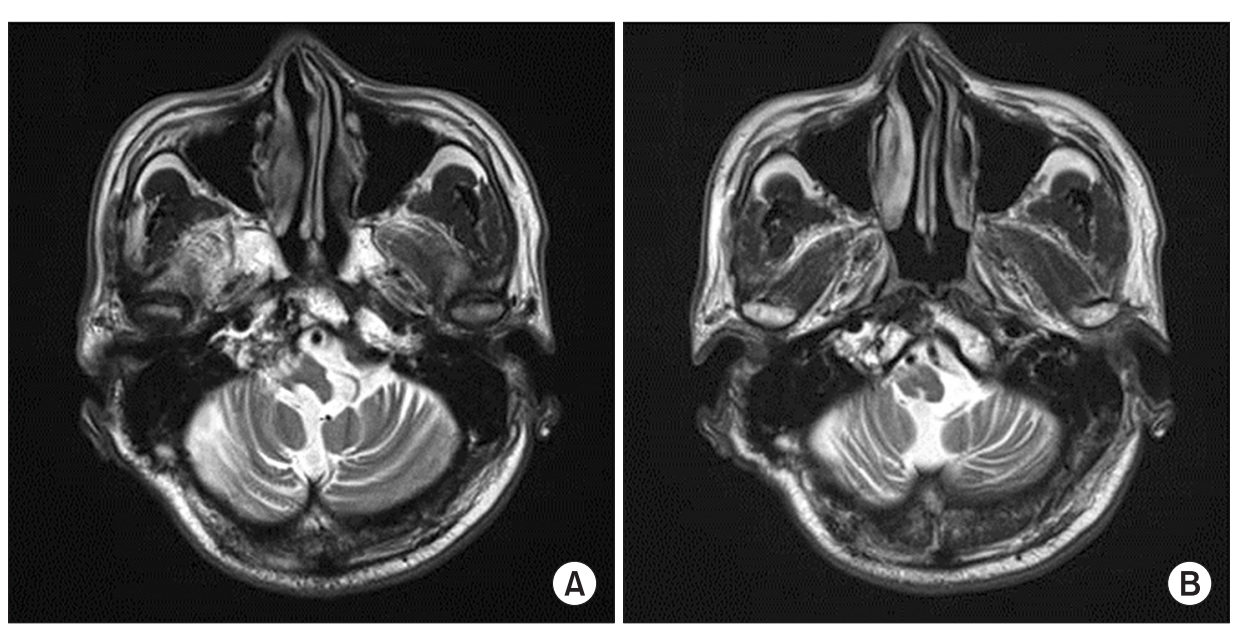

Fig. 1. (A, B) T2-weighted magnetic resonance imaging of the brain shows atrophy of the right brainstem without tumor relapse. 

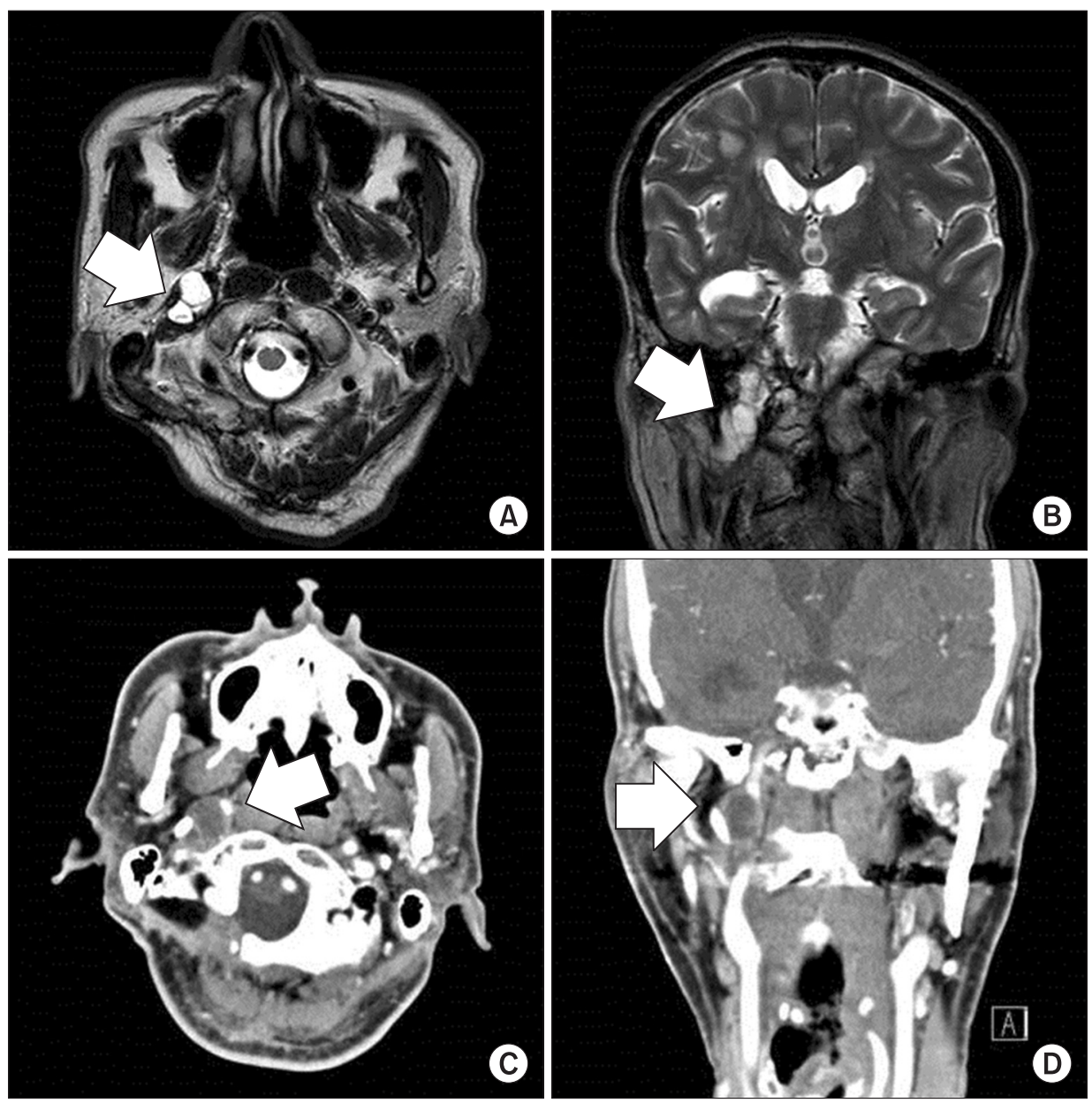

Fig. 2. T2-weighted magnetic resonance imaging of the brain $(\mathrm{A}$, axial view; $\mathrm{B}$, coronal view) shows a $1.9 \mathrm{~cm} \times 1.4 \mathrm{~cm}$ multilobular lesion (arrow) with an intense signal and heterogeneous enhancement of the right clivus, jugular foramen, and hypoglossal canal. Computed tomography of the paranasal sinuses (C, axial view; $\mathrm{D}$, coronal view) shows a mass (arrow) with soft tissue attenuation involving the right petroclival region.

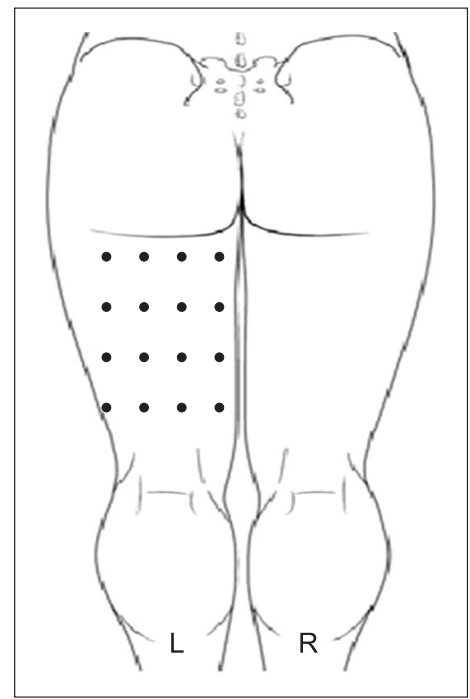

Fig. 3. Schematic representation of botulinum toxin type A injection in the posterior aspect of the left thigh using a grid pattern (16 points). with central pain have physical and cognitive impairments related to the CNS injury and may be less tolerant of medications that cause dizziness, ataxia, confusion, or sedation [1]. Our patient was unable to take a sufficient dose of medications or use neuromodulation techniques, due to side effects. Fortunately, the subcutaneous BTXA injection had sufficient analgesic effect on his central neuropathic pain originating from a brain tumor.

Initially, intramuscular BTX-A injections were used to treat movement disorders, such as spasticity or dystonia. Many recent clinical studies have suggested that subcutaneous BTX-A injection has an analgesic effect on peripheral neuropathic pain, such as post-herpetic neuralgia, diabetic neuropathy, trigeminal neuralgia, and post-traumatic neuralgia $[2,3]$. In addition, two case reports and two randomized controlled studies indicate that BTXA may reduce chronic neuropathic pain in patients with spinal cord injury [4]. In most studies of BTX-A effect on neuropathic pain, BTX-A was administered subcutane- 
ously. The fractioned dosage varied between 2.5 and 7.5 $\mathrm{U} / \mathrm{cm}^{2}$ of the painful surface area, and the total dosage varied between 100 and $200 \mathrm{U}$ [2-4]. We chose the subcutaneous injection of a total of $100 \mathrm{U}$ into the most painful area of the posterior thigh in this case.

Activation of sensory unmyelinated neurons by noxious stimuli evokes the release of neuropeptides (glutamate, substance $\mathrm{P}$, and calcitonin gene-related peptides), causing local edema, hyperemia, and erythema. Since these inflammatory signs depend on the function and the integrity of peripheral sensory nervous system, the response has been termed neurogenic inflammation. The proposed mechanism of analgesic effect of BTX-A is the inhibition of neurogenic inflammation and peripheral sensitization by the attenuation of the release of neuropeptides from nociceptive sensory neurons via peripheral SNAP-25 cleavage. In addition, BTX-A may decrease the delivery to the neuron cell membrane of the transient receptor potential vanilloid-1, which intensifies the excitability of nociceptors. Blocking the peripheral sensitization would reduce the transmission of nociceptive signals to the CNS and also reduce the release of substance P and glutamate in the spinal cord, consequentially reducing the central sensitization $[5,6]$. Reduction of pain intensity, secondary hyperalgesia, flare area, blood flow, and skin temperature following BTX-A treatment of human skin has been described [7]. Contrarily, the absence of an association between anti-inflammatory and antinociceptive effects of BTX-A has been reported. In carrageenan induced inflammation models, BTX-A reduced the mechanical hyperalgesia, but had no effect in local tissue inflammatory edema [8]. In another study, pain was reduced only if capsaicin was administered to the area pretreated with BTX-A in human skin. Neurogenic inflammation, including flare and vasodilation, was inhibited by BTX-A when capsaicin was applied to either the same or adjacent area treated by BTX-A [9].

Evidence for central actions of BTX-A in pain modulation involving the axonal transport of BTX-A from periphery to CNS has been reported. In a sciatic nerve injured mouse model, BTX-A was administered subcutaneously into the hindpaw plantar surface. Immunofluorescence analysis showed the presence of cleaved SNAP-25 in tissues along the nociceptive pathway from the peripheral nerve endings to the spinal cord [10]. In addition, a bilateral anti-nociceptive effect after unilateral injection of
BTX-A was observed in a paclitaxel-induced neuropathy model [8]. The collective observations indicate that subcutaneously injected BTX-A may directly inhibit primary sensory fibers, leading to a reduction of peripheral and central sensitization, with BTX-A transported retrogradely within the axonal compartment to the CNS.

In this case, BTX-A had a significant analgesic effect on the neuropathic pain induced by a brain tumor without apparent side effects. Randomized controlled trials are needed to determine the effective dosage and treatment interval of BTX-A for neuropathic pain in patients with brain tumors. Although the detailed mechanism of the BTX-A-induced pain relief is not fully understood, we believe that BTX-A may be helpful for the management of neuropathic pain results from structural brain lesion, such as brain tumor or stroke, leading to functional abnormalities in the nervous system. Subcutaneous BTX-A would be a useful addition for the treatment of neuropathic pain related to brain tumor.

\section{CONFLICT OF INTEREST}

No potential conflict of interest relevant to this article was reported.

\section{REFERENCES}

1. Watson JC, Sandroni P. Central neuropathic pain syndromes. Mayo Clin Proc 2016;91:372-85.

2. Liu HT, Tsai SK, Kao MC, Hu JS. Botulinum toxin A relieved neuropathic pain in a case of post-herpetic neuralgia. Pain Med 2006;7:89-91.

3. Oh HM, Chung ME. Botulinum toxin for neuropathic pain: a review of the literature. Toxins (Basel) 2015;7: 3127-54.

4. Han ZA, Song DH, Oh HM, Chung ME. Botulinum toxin type A for neuropathic pain in patients with spinal cord injury. Ann Neurol 2016;79:569-78.

5. Cui M, Khanijou S, Rubino J, Aoki KR. Subcutaneous administration of botulinum toxin A reduces formalin-induced pain. Pain 2004;107:125-33.

6. Aoki KR. Review of a proposed mechanism for the antinociceptive action of botulinum toxin type A. Neurotoxicology 2005;26:785-93.

7. Gazerani P, Pedersen NS, Staahl C, Drewes AM, Arendt-Nielsen L. Subcutaneous botulinum toxin type A 
reduces capsaicin-induced trigeminal pain and vasomotor reactions in human skin. Pain 2009;141:60-9.

8. Favre-Guilmard C, Auguet M, Chabrier PE. Different antinociceptive effects of botulinum toxin type A in inflammatory and peripheral polyneuropathic rat models. Eur J Pharmacol 2009;617:48-53.

9. Tugnoli V, Capone JG, Eleopra R, Quatrale R, Sensi $\mathrm{M}$, Gastaldo E, et al. Botulinum toxin type A reduces capsaicin-evoked pain and neurogenic vasodilatation in human skin. Pain 2007;130:76-83.

10. Marinelli S, Vacca V, Ricordy R, Uggenti C, Tata AM, Luvisetto $S$, et al. The analgesic effect on neuropathic pain of retrogradely transported botulinum neurotoxin A involves Schwann cells and astrocytes. PLoS One 2012;7:e47977. 